\title{
PALEO
}

Revue d'archéologie préhistorique

$27 \mid 2016$

Varia

\section{Le bison de La Grèze (Marquay, Dordogne, France) sous le microscope}

The bison of La Grèze cave (Marquay, Dordogne, France) under the microscope

\section{Lydia Zotkina}

\section{(2) OpenEdition}

\section{Journals}

Édition électronique

URL : http://journals.openedition.org/paleo/3170

DOI : $10.4000 /$ paleo.3170

ISSN : 2101-0420

Éditeur

SAMRA

\section{Édition imprimée}

Date de publication : 30 décembre 2016

Pagination : 307-320

ISSN : 1145-3370

\section{Référence électronique}

Lydia Zotkina, « Le bison de La Grèze (Marquay, Dordogne, France) sous le microscope », PALEO [En

ligne], 27 | 2016, mis en ligne le 01 juin 2018, consulté le 07 juillet 2020. URL : http://

journals.openedition.org/paleo/3170; DOI : https://doi.org/10.4000/paleo.3170

\section{(c) (i) $\odot \odot$}

PALEO est mis à disposition selon les termes de la licence Creative Commons Attribution - Pas d'Utilisation Commerciale - Pas de Modification 4.0 International. 


\title{
Le bison de La Grèze (Marquay, Dordogne, France) sous le microscope
}

\author{
Lydia ZOTKINA ${ }^{(a)}$
}

Résumé : S.A. Semenov, le fondateur de la discipline, avait donné un cadre large à la tracéologie. Pourtant, en un demisiècle, la plupart des recherches dans ce domaine se focalisèrent sur l'usure des outils lithiques. Quelques travaux pionniers montrèrent néanmoins que l'analyse des marques d'outils pouvait être efficacement appliquée aux études d'art pariétal pour aborder des questions relatives aux techniques, aux chaînes opératoires et à la chronologie des représentations. Un des aspects qui en découle mais qui n'a pas encore été exploré, est celui du rafraîchissement des figures. Les différences de morphologie observées dans les contours gravés ou piquetés d'une composition peuvent être vues comme le résultat d'évènements distincts. Cela avait été le cas avec le Bison de La Grèze, suspecté d'avoir été partiellement repassé à l'époque de sa découverte pour le rendre plus visible. Un analyse détaillée des contours de la représentation, sous microscope binoculaire et à partir de différentes échelles de modèles 3D, montre que le fond blanc et frais observé en plusieurs points de la gravure ne témoigne pas d'une reprise moderne de la figure mais d'un processus d'altération naturelle de la paroi.

Mots-clés : art rupestre, grotte ornée, tracéologie, taphonomie, 3D.

Abstract: The bison of La Grèze cave (Marquay, Dordogne, France) under the microscope. The scope of traceology, as defined by S.A.Semenov who founded the discipline, was broad, but in half a century most of the studies have focused on the use wear of lithic tools. Nonetheless, some pioneer works show that the analysis of tool marks can provide useful data to rock art studies and helps to address questions about the techniques, the "chaîne opératoire» and the chronology of the pictures. A topic which is directly linked but which has not yet been investigated concerns their renewal. Differences in the morphology of the engraved or pecked lines belonging to a same pattern can be seen as the result of distinct events. This has been the case with the bison of La Grèze cave, suspected to have been partly redrawn at the time of its discovery for making it more visible. A close examination of the outline with a binocular microscope and its 3D modeling at different scales show that, despite a fresh and white bottom of some parts of the groove, there is no evidence of secondary work: all the recent features are due to the natural alteration of the wall.

Key-words: rock art, traceology, taphonomy, 3D.

\section{Introduction}

Si S.A. Semenov est reconnu comme l'inventeur de la tracéologie en tant que discipline archéologique (Семенов 1957, Semenov 1964), la définition qui en fut faite en Europe et aux États-Unis fut plus restrictive qu'en Russie. Son champ d'application se limita pendant longtemps à l'usure des tranchants d'outils lithiques taillés, le plus souvent analysés au microscope à fort grossissement, alors que la tracéologie avait été initialement conçue pour prendre en compte non seulement une grande diversité de matériaux mais aussi les traces de fabrication produites par toutes sortes de techniques et par conséquent, le recours à différentes échelles d'observation (Longo et Skakun 2005). Plus largement, tout objet archéologique est potentiellement

(a) Institut d'Archéologie et d'ethnographie, branche sibérienne de l'Académie des Sciences de Russie, 17 perspective de l'Académicien Lavrentiev, 630090 Novosibirsk, Fédération de Russie et Université d'état de Novosibirsk, département d'archéologie et d'ethnographie, 2 rue Pirogova, 630090 Novosibirsk, Fédération de Russie - lidiazotkina@gmail.com 
sujet à une lecture tracéologique puisqu'il est lui-même la trace matérielle d'une action humaine.

D'ailleurs, l'approche tracéologique est implicite ou latente dans des études qui ne la revendiquent pas comme telle ou bien la qualifient de technologique, en particulier dans le milieu souterrain (traces d'extraction minière, ex. Arles et al. 2013 ; techniques graphiques, ex. Tosello et Fritz 2005, D’Errico et al. 2016), la validité de la démarche dépendant fondamentalement de la pertinence des référentiels mobilisés.

L'étude de l'art préhistorique offre un champ d'application propice à la tracéologie telle que la concevait S.A. Semenov, que ce soit sur support mobilier ou sur parois. Outre la reconnaissance des techniques, elle se prête à l'étude de la reconstitution des chaînes opératoires, c'est-à-dire à la reconstitution du déroulement, au cours du temps, des actions ayant marqué le support peint, raclé, gravé, piqueté ou sculpté.

La chronologie relative des traits constituant les figures, et celle des figures formant les compositions, font partie du registre des questions susceptibles d'être abordées lors de l'analyse des représentations. Les principaux critères considérés sont la chronologie de la superposition des traits et leur homogénéité, dont sont déduits l'ordre des tracés et leur appartenance à un même ensemble, la synchronie technique contribuant à la définition d'unités graphiques. Cette approche est nécessaire pour démêler les palimpsestes (p. ex. Aubry, Santos et Luis 2014), fréquents dans l'art paléolithique, plus particulièrement sur les supports mobiliers. Ceux-ci, néanmoins, rapidement enfouis du fait de leurs petites dimensions, ont été peu propices à des réemplois prolongés, à la différence des parois et des surfaces rocheuses potentiellement accessibles durant des millénaires, parfois jusqu'aux temps présents. Des mêmes lieux ont pu être occupés à différentes époques de la Préhistoire et leurs compositions graphiques complétées ou transformées par des artistes employant des codes assez semblables, à notre échelle de perception, comme dans la grotte Chauvet (Clottes 2010). Ils ont pu aussi être fréquentés bien postérieurement par des individus inscrivant leurs marques pour d'autres motifs, que ce soit des graffitis attestant de leur passage, facilement distinguables tout comme, par exemple, les symboles chrétiens surajoutés aux figures "païennes » (ex. Hinout 1998) ou, au contraire, des rafraîchissements moins évidents à discerner, destinés à faire revivre les représentations, tel que cela est connu ethnographiquement (Miklashevich 2008 ; Миклашевич 2011), ou à les rendre plus visibles lors de leur découverte ou de leur exposition au public. Les combinaisons sont multiples et une analyse stylistique n'est pas toujours suffisante pour distinguer les œuvres initiales des reprises ou des ajouts postérieurs.

Dans tous les cas, la question du temps demeure au centre des préoccupations, qu'elle concerne la relation entre deux traits, entre différentes parties d'une figure, entre les figures d'un ensemble ou entre les compositions graphiques d'un même espace (de la paroi au site). Dans certains contextes, une datation isotopique peut être opérée directement sur les figures lorsqu'elles comportent des pigments organiques, ou sur les dépôts carbonatés qui les ont scellées (ex. Genty et al. 2011 ; Benson et al. 2013). Mais le plus souvent, les indices de datation sont indirects et s'appliquent à l'ensemble d'une composition lorsque celle-ci est au contact d'un dépôt stratigraphique lui-même daté de diverses façons (ex. Aubry et Sampaio 2008). Aussi, c'est à l'échelle même des tracés qu'il faut rechercher des indices supplémentaires. II y a là un champ d'investigation brillamment illustré par les travaux pionniers de D’Errico, Sacchi et Vanhaeren (2002) et de Gyria et Devlet (Гиря еt Дэвлет 2010), à partir de la signature des outils de gravure et de piquetage, et plus particulièrement d'une distinction entre les formes lithiques et métalliques, ce qui peut être parfois très utile pour préciser la chronologie relative.

Parallèlement aux référentiels de comparaison, l'efficacité de l'analyse tracéologique repose sur les techniques d'observation et d'enregistrement. Celles-ci ont considérablement évolué ces dernières années grâce à l'imagerie 3D devenue plus accessible et utilisable sur le terrain à des échelles compatibles avec la lecture des traces les plus fines (Plisson et Zotkina 2015). Cet aspect est particulièrement important dans le cas des pétroglyphes car le relief des tracés ou des formes rend nécessaire la prise en compte de la $3^{\circ}$ dimension. Jusqu'alors, le recours à des empreintes (silicone ou autre) était inévitable pour procéder à des mesures et des relevés de profils (D'Errico, Sacchi et Vanhaeren 2002 Гиря еt Дэвлет 2010), avec tous les risques de pollution et d'arrachement que cela représentait pour les surfaces. Ce procédé, dorénavant proscrit, est aujourd'hui avantageusement remplacé par la photogrammétrie.

La démarche tracéologique élaborée et mise en œuvre sur des sites à pétroglyphes de plein air gravés et piquetés (D’Errico, Sacchi et Vanhaeren op.cit. ; Gyria \& Devlet op.cit. ; Zotkina 2012 ; Зоткина 2013 ; Черемисин et al. 2013 ; Zotkina et al. 2014 n'avait pas encore montré son utilité en milieu souterrain où les figures peintes concentrent l'essentiel des analyses. À titre de démonstration, nous l'avons appliquée à la question des reprises de figure, à partir de l'exemple préliminaire du bison de La Grèze.

\section{Étude de cas}

La grotte-abri de La Grèze est une petite cavité dans la vallée de la Grande Beune, en contrebas du chemin vicinal reliant la RD 48, sur la commune de Marquay en Dordogne (Patriarche 24255 0003). Elle est connue pour la figuration gravée d'un bison de $60 \mathrm{~cm}$ sur $40 \mathrm{~cm}$ (fig. 1), bien conservée du fait de sa situation dans un renfoncement concave de la paroi la protégeant de l'érosion, à la différence d'autres représentations animales dont ne subsistent plus que les parties basses.

La cavité et ses gravures ont été étudiées pendant plus d'un siècle, à partir de 1904 (Capitan, Breuil et Ampoulange 1904a, 1904b) et plusieurs chercheurs s'y succédèrent 

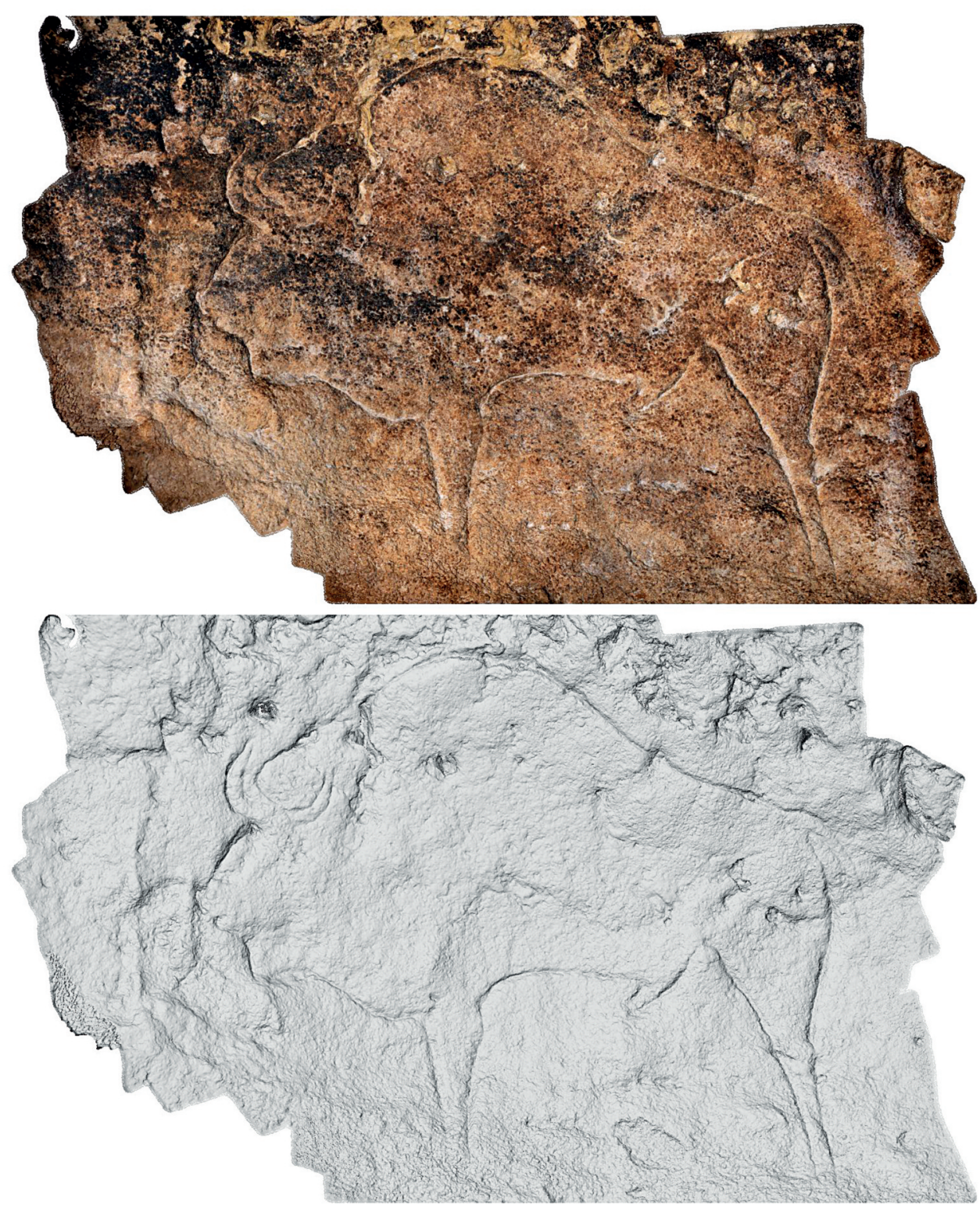

Figure 1 - Vue générale 3D du bison de La Grèze, réalisée par photogrammétrie.

Projection orthographique, avec et sans texture colorée. Nuage de points ultra dense (47 millions de points) (Photo H. Plisson).

Figure 1 - Global 3D view of the bison of La Grèze cave, made by photogrammetry. Orthographic projection, with and without colored texture. Very dense points clouds (47 million vertices) (Picture H. Plisson).

(Ampoulange et Pintaud 1955 ; Aujoulat 1984 ; Breuil 1952 ; Cretin 2015 ; Delluc et Delluc 1991, 1992 ; Delluc et Bouvier 1984 ; Jaubert 2008 ; Peyrony 1949 ; Roussot 1965 ; Sonneville-Bordes 1960, 1965 ; Vidal 1967).

Grâce aux techniques modernes, nous pouvons aujourd'hui prolonger leurs travaux et répondre à quelquesunes de leurs interrogations.

\section{1 - La figuration du bison a-t-elle été surgravée ?}

La couleur claire des traits du bison attire l'attention et l'on supposa que ce fait pouvait être expliqué par un rafraîchissement de la figure : " II n'est pas impossible que les traits aient été surgravés récemment avec un outil pointu, car le calcaire est beaucoup plus clair au fond des traits que sur leurs berges »(Delluc et Delluc 1991 p. 238) ; « ... accessoirement le bison célèbre présente quelques traces de moulage et peut-être son trait de contour a-t-il été discrètement surgravé " (Delluc et Delluc 1991 - p. 245)

\section{2 - Technique : piquetage et/ou gravure profonde?}

Dans tous les ouvrages consacrés à La Grèze, les chercheurs mentionnent la figuration du bison comme étant gravée (Peyrony 1949 ; Breuil 1952 ; Aujoulat 1984 ; Delluc et Delluc 1991). " La gravure est réalisée par des traits profonds à section angulaire dont le fond est plus ou moins émoussé. Les bords sont souvent arrondis mais ce modelage du bord interne peut être lié plus à l'érosion qu'à l'origine humaine » (Delluc et Delluc 1991 - p. 238). 
Toutefois, Rigaud (1984 - p. 278) mentionne une autre technique qui peut également avoir été utilisée : « Les autres traits sont quelques traits fins à section angulaire, les traits vigoureux obtenus par piquetage et quelques traits larges, vestiges probables de traits vigoureusement gravés ».

La technique du piquetage peut être considérée comme une des explications de l'irrégularité des tracés du bison.

\section{3 - L'altération du support modifie-t-elle les gravures?}

La première chose à considérer quand on aborde les traces techniques dans l'art rupestre, et de façon plus générale en tracéologie, est l'influence de l'altération naturelle des surfaces sur les traits et sur les caractères qui sont a priori vus comme étant diagnostiques, c'est-à-dire ressemblant à des marques d'outil (Kervazo et al. 2010). Sans compréhension des effets et des mécanismes de l'altération, le risque de tirer de fausses conclusions n'est pas négligeable.

C'est pourquoi cette question doit être première dans le cadre d'une telle recherche.

\section{Techniques, méthode, et équipement}

La présente recherche est centrée sur les aspects tracéologiques. Elle change l'échelle des observations et permet d'approfondir la compréhension des mécanismes de transformation des reliefs de la surface rocheuse.

Nos observations ont été faites avec une loupe binoculaire WILD M1A (x7, x14). La distance de travail de ce microscope (une vingtaine de centimètres) est importante et permet d'éviter le contact accidentel avec la surface examinée. Sa légèreté permet de travailler sans pied (fig. 2).

Pour l'enregistrement et la comparaison des reliefs, nous avons eu recours à la photogrammétrie (Plets et al. 2012 ; Robert et al. 2012 ; Belarbi et al. 2012 ; Cassen et al. 2012 ; Plisson 2015). Les prises de vues ont été réalisées au moyen d'un boîtier photographique Nikon 750D (capteur 35,9 × $24 \mathrm{~mm}$, résolution 24,93 million pixels), pourvu d'un objectif AF-S Micro NIKKOR $60 \mathrm{~mm}$ f/2.8G et d'un flash annulaire. Les modèles sont générés puis traités avec les logiciels Agisoft Photoscan, MeshLab et GeoMagic, à partir desquels nous extrayons des coupes transversales des tracés.

Nous avons également réalisé des macrophotographies de détails caractéristiques de la figure avec un boîtier Canon $1000 \mathrm{D}$ de format $22 \times 15 \mathrm{~mm}$ pourvu d'un objectif Canon EF$\mathrm{S} 60 \mathrm{~mm}$ f/2.8 Macro USM et de bagues d'extension, fixé sur un rail à crémaillère et un robuste tripode. Les séquences de clichés ont été pilotées à partir d'un ordinateur, puis compilées avec le logiciel Helicon Focus pour obtenir des vues de haute résolution en hyper focus.

Pendant les observations et les enregistrements, nous avons utilisé un éclairage à leds Aurora Max 5600 dont

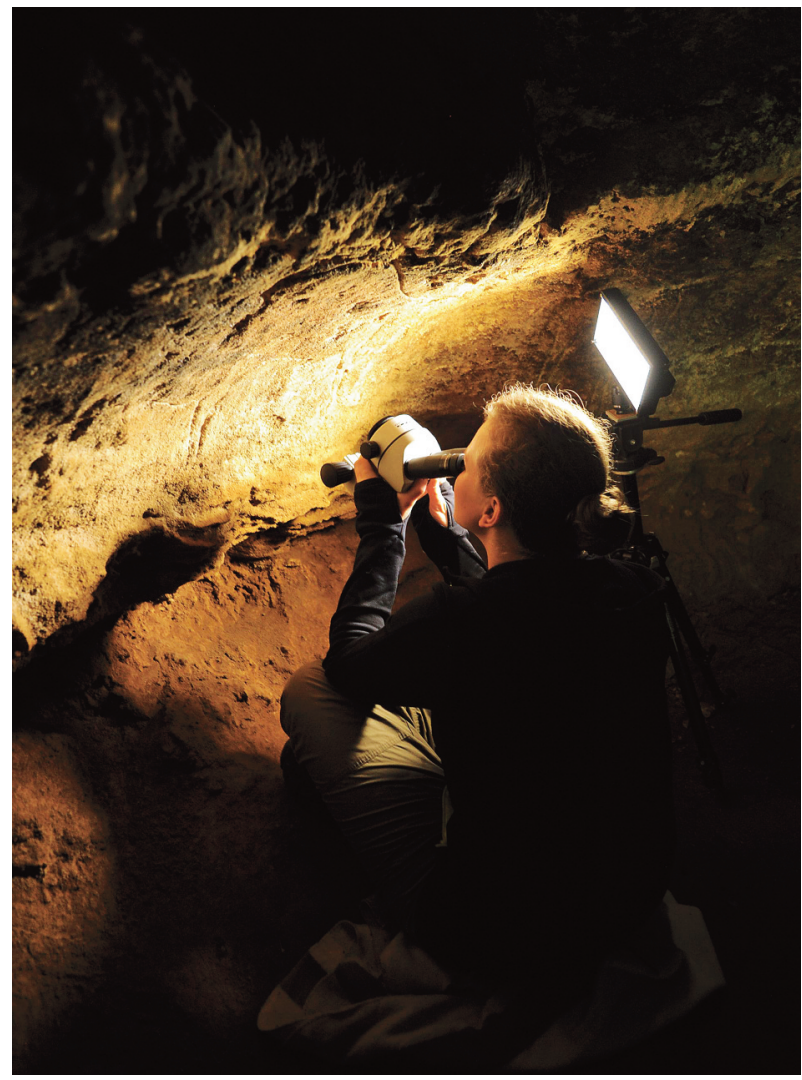

Figure 2 - Dispositif d'observation à la binoculaire in situ (Photo H. Plisson).

Figure 2 - In situ examination (Picture H. Plisson).

l'intensité lumineuse et la température de couleur pouvaient être ajustées.

Les références expérimentales pour comparaison sont celles développées dans la thèse récente de l'auteur (Zotkina 2013), établies sur différents terrains, et enrichies par des exemples archéologiques et ethnographiques relatifs à différentes techniques (Plisson et Zotkina 2015), avec des outils lithiques et métalliques. Les observations faites à La Grèze ont été complétées par des tests (fig. 7) sur des petits blocs tombés à l'extérieur, au pied de l'abri.

Mais il faut considérer que les moyens mis en œuvre ici ne sont pas toujours possibles dans la configuration des grottes ornées (difficultés d'éclairage, difficultés pour s'approcher des panneaux, finesse de certaines gravures, étendue des surfaces ornées, etc).

\section{Rainurage, piquetage ou altération de la surface?}

Les traits du bison et de la silhouette identifiée comme celle d'un mammouth, sur la même paroi, sont très irréguliers et parfois très profonds. Certaines portions de surface des deux figurations sont très alvéolaires, ce qui permet d'émettre l'hypothèse que ces traits auraient été exécutés par la technique du piquetage. B. et G. Delluc indiquent une 
variation assez visible de la profondeur (entre 2 et $7 \mathrm{~mm}$ ) et de la largeur des traits du bison (entre 3 et $12 \mathrm{~mm}$ ) (Delluc et Delluc 1991 - p. 238). Les tracés sont parfois décrits comme ayant une section " recticurviligne » (op. cit., fig. 168). La technique de la photogrammétrie permet de restituer précisément leur morphologie (Plisson et Zotkina 2015). Sur nos modèles 3D sont visibles des sections qu'on ne peut qualifier de typiques pour des traits de gravure (D'Errico, Sacchi et Vanhaeren 2002), lesquels ont habituellement plutôt un profil régulier plus ou moins en $\mathrm{V}$ (fig. 3 a-g).

II est cependant évident que le support est fragile et assez altéré, comme l'avait déjà évoqué les Delluc (1991 p. 235).

Lors de nos observations à la loupe binoculaire nous avons constaté les caractéristiques suivantes :

La conservation des marques d'outil dans les figurations d'autres grottes de la région (par exemple Combarelles I et II, Font-de-Gaume) peut être bien meilleure qu'à La Grèze ; on y observe des signatures beaucoup plus visibles et parlantes. En effet, en bordure du tracé principal large et profond du bison, nous n'avons aucune trace plus fine rendant compte des spécificités de la partie active de l'outil, ni de tracé parasite trahissant des écarts de geste. L'altération importante ne permet pas d'aller au-delà du relevé des profils du sillon. Cette comparaison nous conduit à penser que l'état de conservation non seulement du support lui-même mais aussi des tracés préhistoriques sur les parois de La Grèze est peu propice à une recherche tracéologique approfondie.

La comparaison de certains tracés du bison (fig. 3, a, f) avec les parties naturellement altérées qui n'ont pas une morphologie de trait gravé mais qui leur ressemble au niveau du contour (fig. $3, \mathrm{~d}, \mathrm{e}$ ) montre des similitudes qui laissent penser que des zones considérées comme piquetées seraient en fait altérées (fig. 3)

C'est pourquoi nous supposons que nous n'observons pas là des marques d'outil mais des formes produites par l'altération (fig. 3, 4).

Nous avons la chance d'avoir quelques traits plus parlants comme ceux du dos du bison (fig. $4 \mathrm{c}$-h) : le tracé y est assez étroit et les bords presque réguliers, ce qui ne peut être obtenu par piquetage. Nous remarquons aussi une ligne au fond du trait qui n'est pas vraiment une empreinte de partie active, car la surface est tout de même assez altérée, mais qui montre néanmoins le relief général du tracé. Cette ligne-là évoque un geste qui ressemble plutôt à celui du rainurage, donc à de la gravure profonde. Nous sommes dans la partie de la figuration où la surface est plutôt mieux conservée par rapport aux autres zones. Surtout, il nous semble que le geste n'y a pas été très vigoureux.

Un autre indice de la technique de gravure remarquable au niveau des cornes nous semble important à décrire : il est visible (fig. 5), lorsque les traits changent d'orientation de façon assez brusque, certainement aux endroits où la matière a résisté à l'outil lors de l'exécution du tracé. En effet, en franchissant des parties plus dures de la matière l'outil a dévié. C'est très typique pour la technique de la gravure, car l'outil suit la surface en restant presque tout le temps en contact, et il se trouve guidé par la structure de cette matière, ce qui n'est pas du tout le cas avec le piquetage.

Un argument supplémentaire est la dégradation de la représentation du mammouth (fig. 6) située à une cinquantaine de centimètres à gauche, sur la même paroi. On y observe les conséquences de la desquamation ayant presque détruit la figure (fig. $6, \mathrm{a}, \mathrm{b}$ ). On y voit néanmoins quelques traces conservées, par exemple un trait extrêmement étroit et profond avec des bords réguliers, qui se trouve au niveau de la patte (fig. $6 \mathrm{c}$ ). Cela nous permet de dire que l'altération de la surface a élargi les traits et modifié leur configuration.

Nous arrivons donc à la conclusion que la figuration du bison a été exécutée par la technique de la gravure profonde, que l'on peut qualifier de rainurage, mais que l'évolution de la paroi a, par endroits, donné au tracé une apparence piquetée.

\section{Vérification expérimentale}

Six faciès géologiques ont été identifiés dans la grotte (Le Filiâtre in Cretin et al. 2015 - p. 50, fig. 1/23). La plupart des figures, au moins les plus célèbres, sont gravées dans le faciès D «Calcaire blanc pseudo-oolithique ». C'est un matériau assez homogène et tendre, facile à travailler par la technique de la gravure. Pour nos expérimentations, nous avons pris des petits blocs du même faciès (fig. 7). Nous avons testé une méthode de rainurage susceptible de produire un sillon profond comme celui de la silhouette du bison, tel qu'il peut être observé dans les parties les moins altérées du tracé. Nous avons utilisé un éclat de silex local, sur la surface brute à l'humidité ambiante d'un sous-bois de printemps, mais sans addition d'eau ni d'autres composants.

Un sillon profond est obtenu en combinant deux gestes : d'abord un geste vigoureux en répétant le mouvement 4 à 5 fois dans une seule direction, jusqu'à obtenir une rainure assez marquée permettant de guider l'extrémité active de l'outil, puis l'approfondissement par un mouvement de vaet-vient. Lorsque la rainure devient assez profonde, l'outil commence alors à en lisser les bords d'un sillon, qui vont progressivement s'arrondir (fig. 7, a, b). Cela correspond bien aux sections des traits du bison, au niveau du dos (fig. $4 \mathrm{c}, \mathrm{f}$ ) où nous reconnaissions les indices caractéristiques du rainurage. Le contour de sa tête est plus dégagé, ce qui a impliqué d'incliner l'outil pour réduire le bord extérieur de la rainure.

\section{Les traits plus clairs}

Comme cela a été cité plus haut, des chercheurs ont posé la question du rafraîchissement du tracé du bison (Delluc et Delluc 1991 - p. 238, 245). Lors de nos observations, nous n'avons identifié que deux zones, de dimension réduite, où 


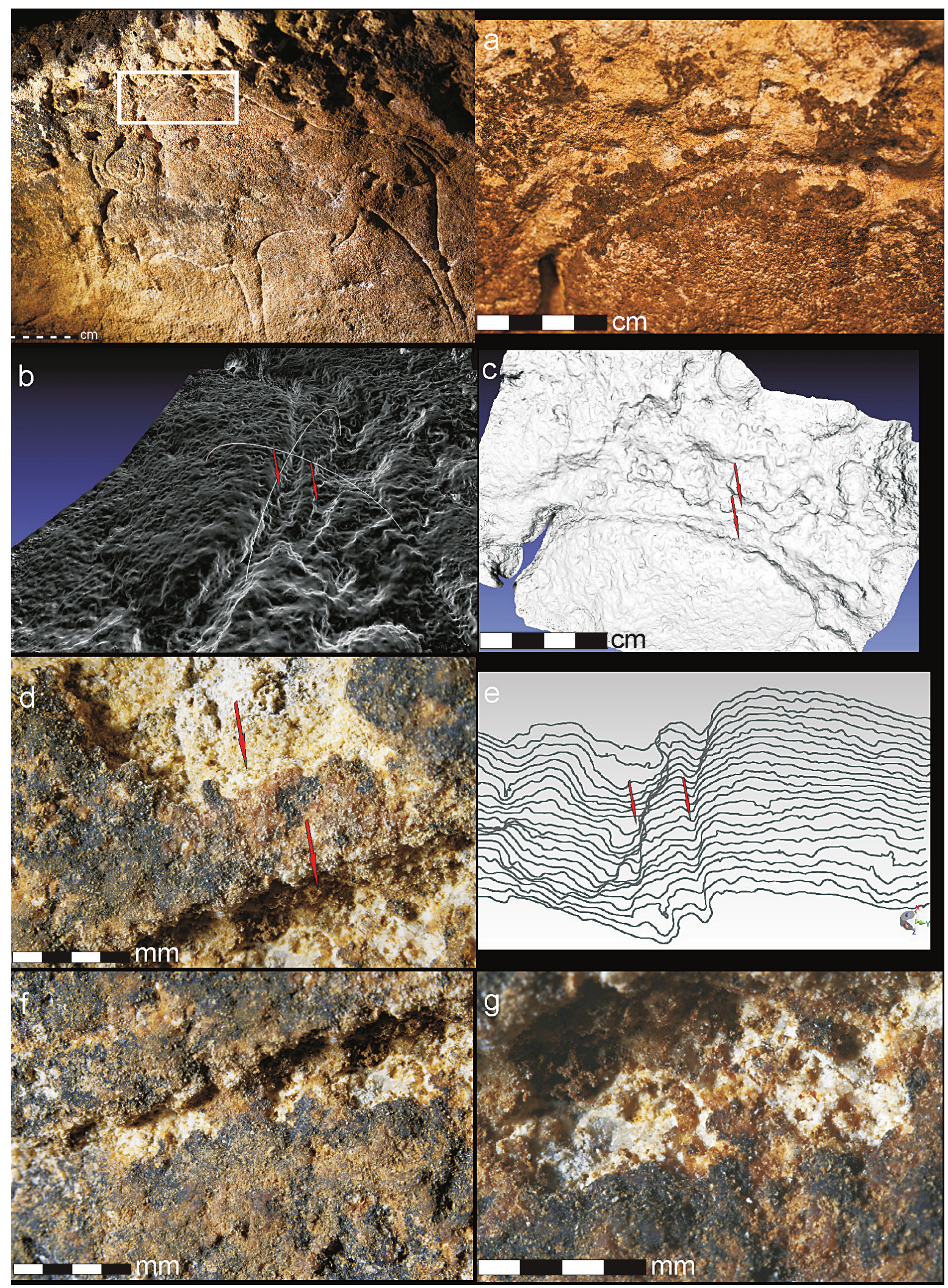

Figure 3 - Le tracé irrégulier de la bosse du bison : vue générale de la figuration avec localisation de la zone considérée; a - photo de la zone du tracé aux bords très irréguliers ; b,c-restitution $3 D$ de la zone irrégulière ; $d$-macrophotographie de la ligne de bosse du bison (au-dessus) et de la zone altérée naturellement (au-dessus) ; e - sections des deux zones (altération naturelle et tracé) ; $f, g$ macrophotographies de la ligne de bosse du bison (Photo L. Zotkina).

Figure 3 - Irregular outline of the bison hump: global view of the bison with the area under focus; a - outline of the hump with very irregular edges; $b, c-3 D$ model of the area; $d$ - macrophotography of the hump outline (lower side) and of the natural alteration of the wall (upper side); $e$ - cross sections of both zones; $f, g$ - macrophotographic details of the hump outline (Picture L. Zotkina). 


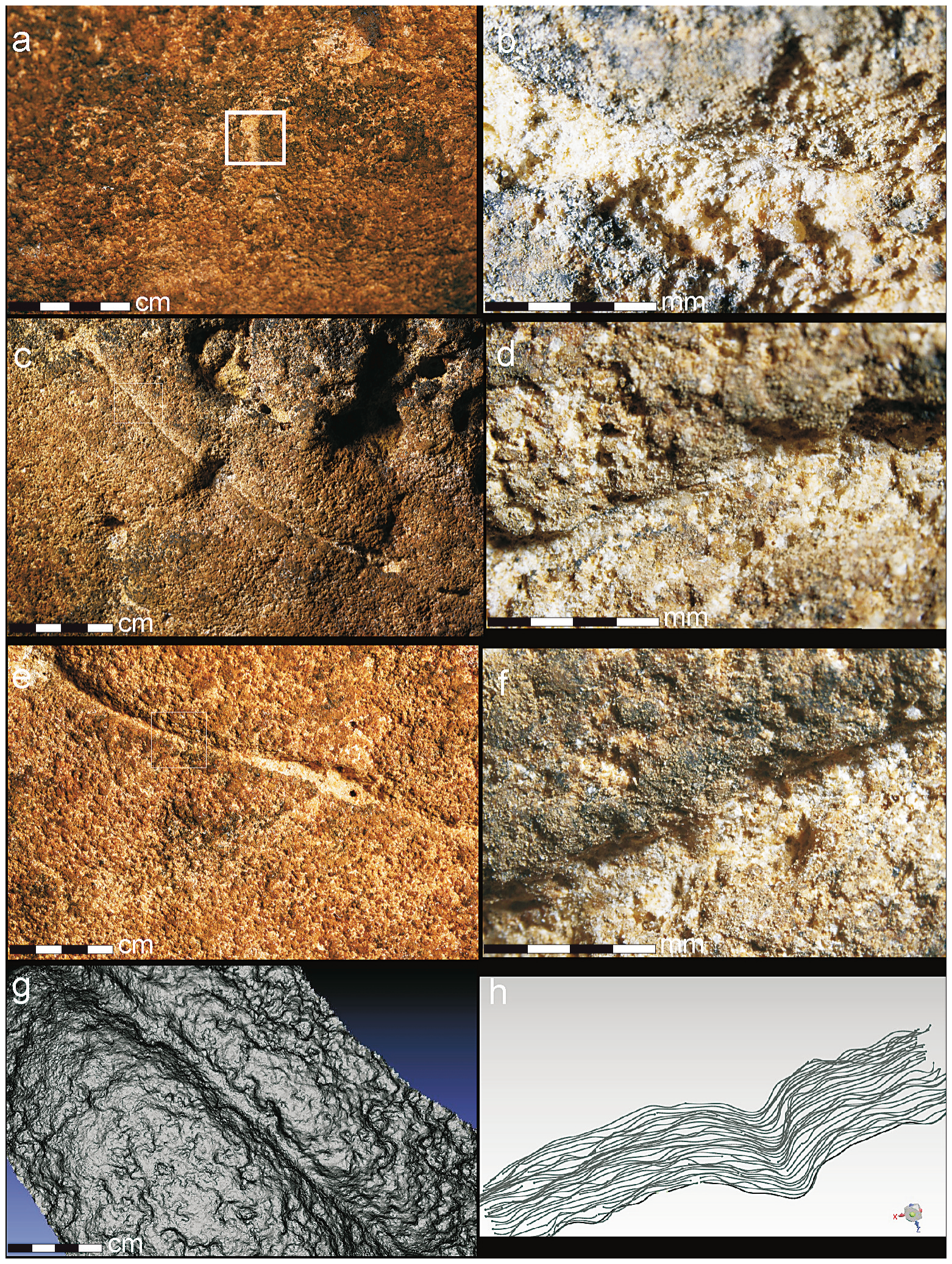

Figure 4 - Gravure et marques naturelles ou accidentelles : $a, b$-exemple de trait accidentel ou naturel au niveau du flanc du bison; $c$, $d$, $e, f$-zones de la ligne de dos du bison à section en $V$ et aux bords réguliers ; $g, h$ - restitution $3 D$ de la zone régulière de la ligne de dos du bison (Photo L. Zotkina).

Figure 4 - Engraving and natural or accidental traces: $a, b$ - samples of a natural of accidental linear mark on the flank of the bison; $c, d, e, f$ - zones of back outline of the bison with regular edges and V profile; $g, h$ - 3D models of the regular outline (Picture L. Zotkina). 


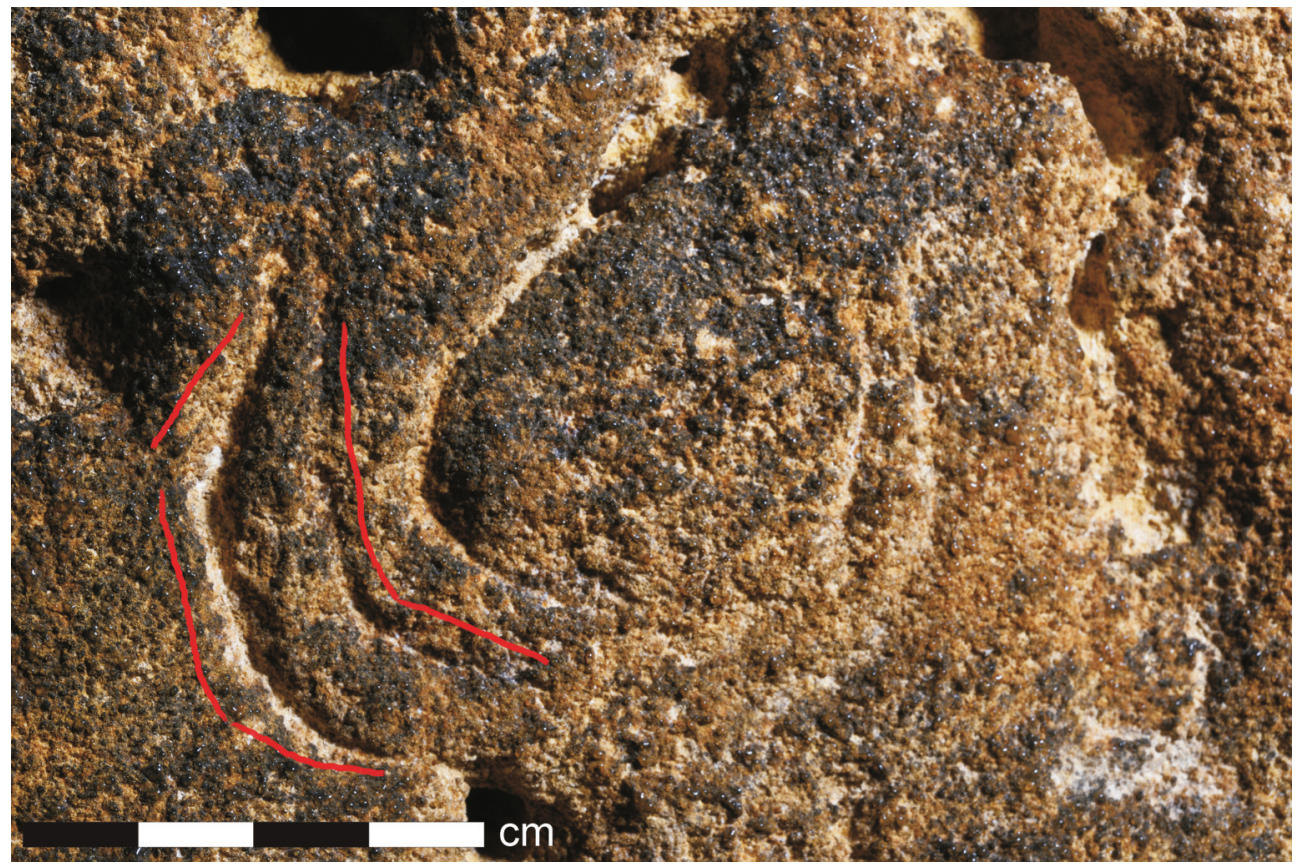

Figure 5 - Cornes du bison : les lignes gravées (les lignes rouges indiquent les endroits où la surface a résisté à la progression de l'outil) (Photo L. Zotkina)

Figure 5 - Bison horns: engraved outlines (red lines underline the parts with an higher resistance to the tool trajectory) (Picture L. Zotkina).

le fond du trait est foncé, c'est-à-dire de la couleur de la paroi : ces deux zones sont situées au niveau de la queue et du dos. Toutes les autres parties de la gravure sont claires.

Pour déterminer si la figure a été surgravée à l'époque contemporaine, deux aspects sont à considérer. Le premier a trait à l'altération naturelle de la zone concernée et sa relation avec le tracé, et le second aux marques qui seraient propres à un outil moderne.

Comme nous l'avons remarqué, avec l'altération, la surface devient très irrégulière et les bords des concavités naturelles sont rugueuses mais ces zones-là sont assez claires. Si l'on compare à celles-ci les sections des zones de la figuration du bison que nous estimons être très altérées et celles qui présentent des caractères représentatifs de la technique de gravure profonde, nous voyons que les premières sont beaucoup plus proches des formes de dégradation que les secondes (fig. 3, fig. 4 a, b, fig. $6 \mathrm{a}, \mathrm{b}$ ). Par endroits, on voit que la rainure foncée et étroite se transforme très graduellement en sillon plus large, beaucoup moins régulier et plus clair ce que nous considérons être la conséquence de l'altération (fig. 4 c, e).

Nos observations à la loupe binoculaire n'ont révélé aucune signature d'outil moderne dans le tracé du bison, pas plus que dans celui du mammouth, ce qui devrait pourtant être le cas si les figures avaient été récemment rafraîchies. Nous avons des exemples de traces contemporaines faites à l'époque des fouilles de la grotte. Sur celui de la figure 8, référencé comme tel par les gestionnaires de la cavité, on voit bien les marques de l'outil qui a attaqué la matière : le fond du trait a été compacté et un peu lissé (fig. 8), et surtout des stries fines, qui soulignent le mouvement, y sont bien visibles (fig. 8). Ces traces se trouvent également sur la paroi opposée à celle du bison mais dont l'état de surface est très similaire. Elles peuvent donc être prises en considération lors de la comparaison des traces techniques et de traces taphonomiques et, par la suite, être incluses dans l'argumentation : rien ne soutient l'hypothèse d'un surlignement moderne de la figuration du bison et du mammouth. L'élargissement du tracé et ses irrégularités sont le fait seul de l'altération naturelle de la paroi.

L'argument selon lequel le fond clair de certaines portions du tracé témoignerait d'une reprise récente n'est finalement pas si déterminant, car c'est là l'une des conséquences de l'altération. Plusieurs plages de desquamation naturelle sont tout aussi éclaircies (fig. 3, fig. 4, a, b, fig. 6, a, b). Le phénomène touche les zones dégradées du bison comme du mammouth. II est actif autant à l'échelle macroscopique, avec un effet bien perceptible, qu'à l'échelle microscopique où une micro-desquamation, quoique moins flagrante, altère aussi inexorablement la surface et donc la patine millénaire qui avait assombri les tracés. Toutefois, la méthode appliquée ici ne nous permet pas de dire à quelle période cette altération s'est produite ni quelle fut son intensité.

Nous pouvons donc conclure que l'apparence actuelle de la figuration du bison de La Grèze ne doit rien à une main récente.

\section{Conclusion}

Si la silhouette du bison de La Grèze fraîchement gravée apparaissait, à l'époque, vraisemblablement plus claire que la paroi, la perception que nous en avons aujourd'hui n'est pas due à la fraîcheur du trait mais à une altération 


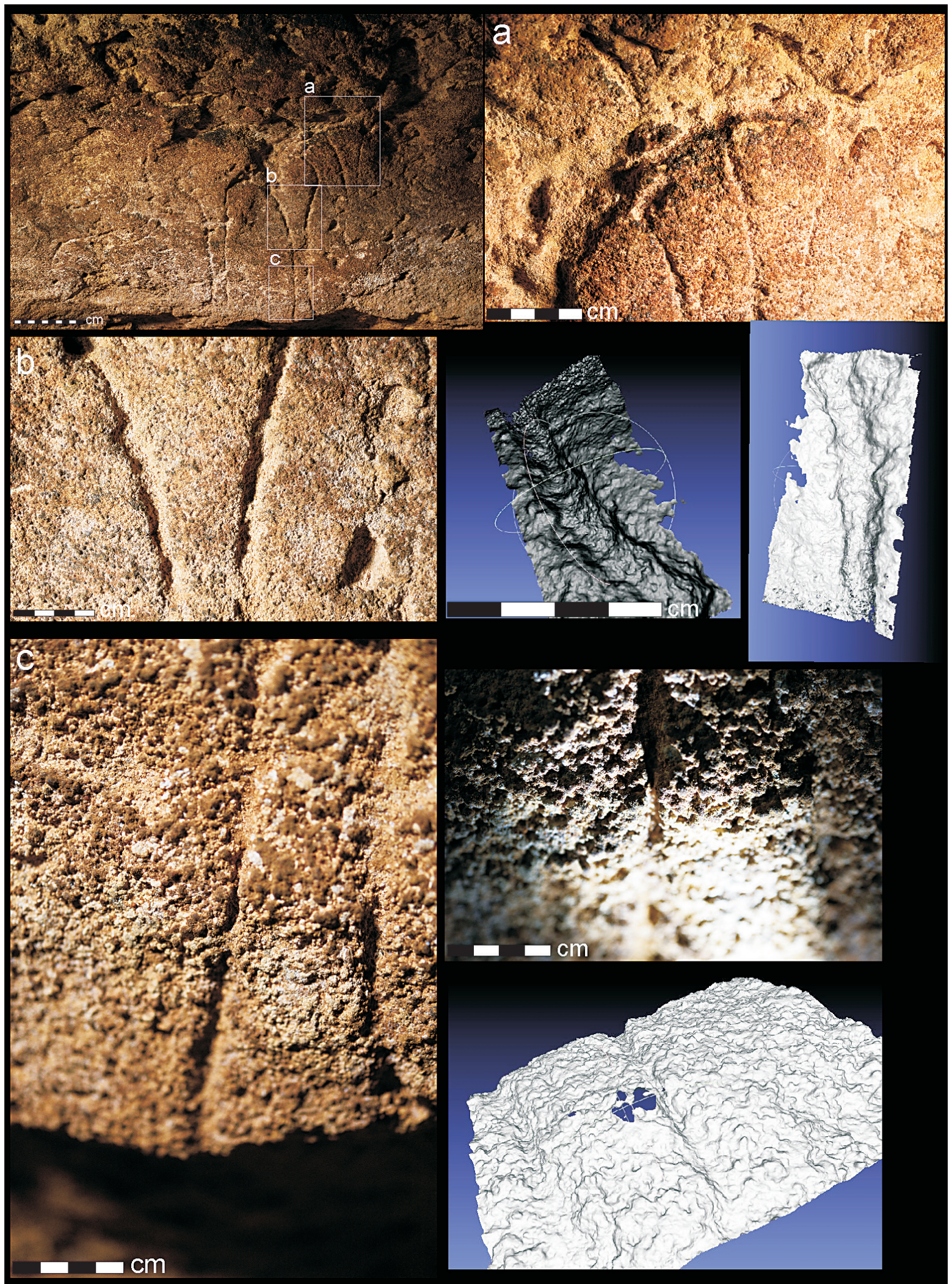

Figure 6 - Figuration de mammouth : a - épaule du mammouth, surface très altérée, irrégulière, $b$ - photo et 3D du tracé large altéré du corps du mammouth ; $c$ - photos et $3 D$ de tracé très fin, recouvert par un concrétionnement grossier (Photo L. Zotkina)

Figure 6 - Engraved mammoth: $a$ - shoulder of the mammoth, very altered and irregular surface; $b$ - photographic view and 3D model of the large altered outline of the body; $c$ - photographic view and 3D model of a fine outline covered by a rough concretion (Picture L. Zotkina). 

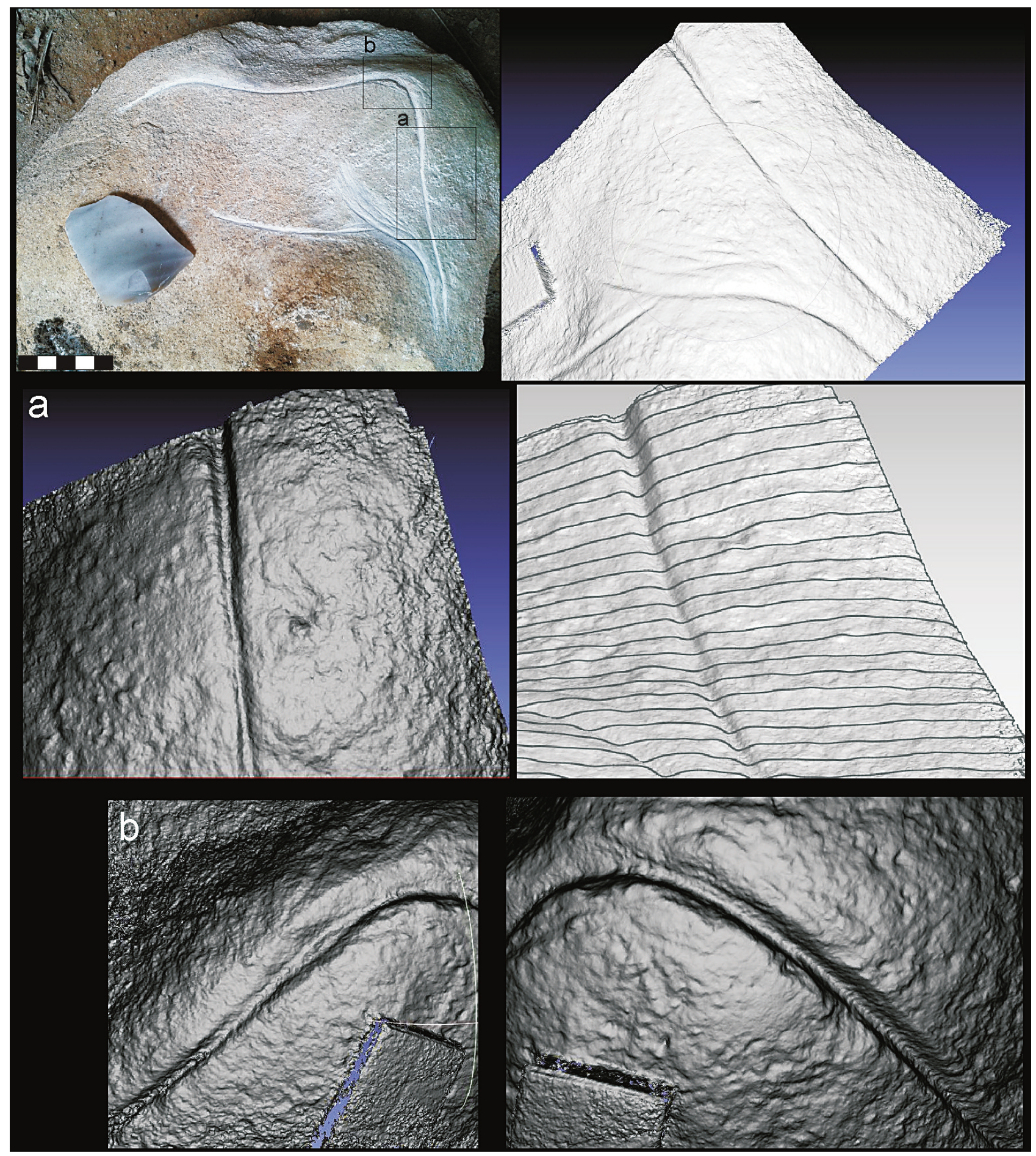

Figure 7 - Gravure expérimentale : $a, b-3 D$ des zones profondément gravées (Photo L. Zotkina).

Figure 7 - Experimental engraving: $a, b-3 D$ model of deep engraved outline (Picture L. Zotkina). 


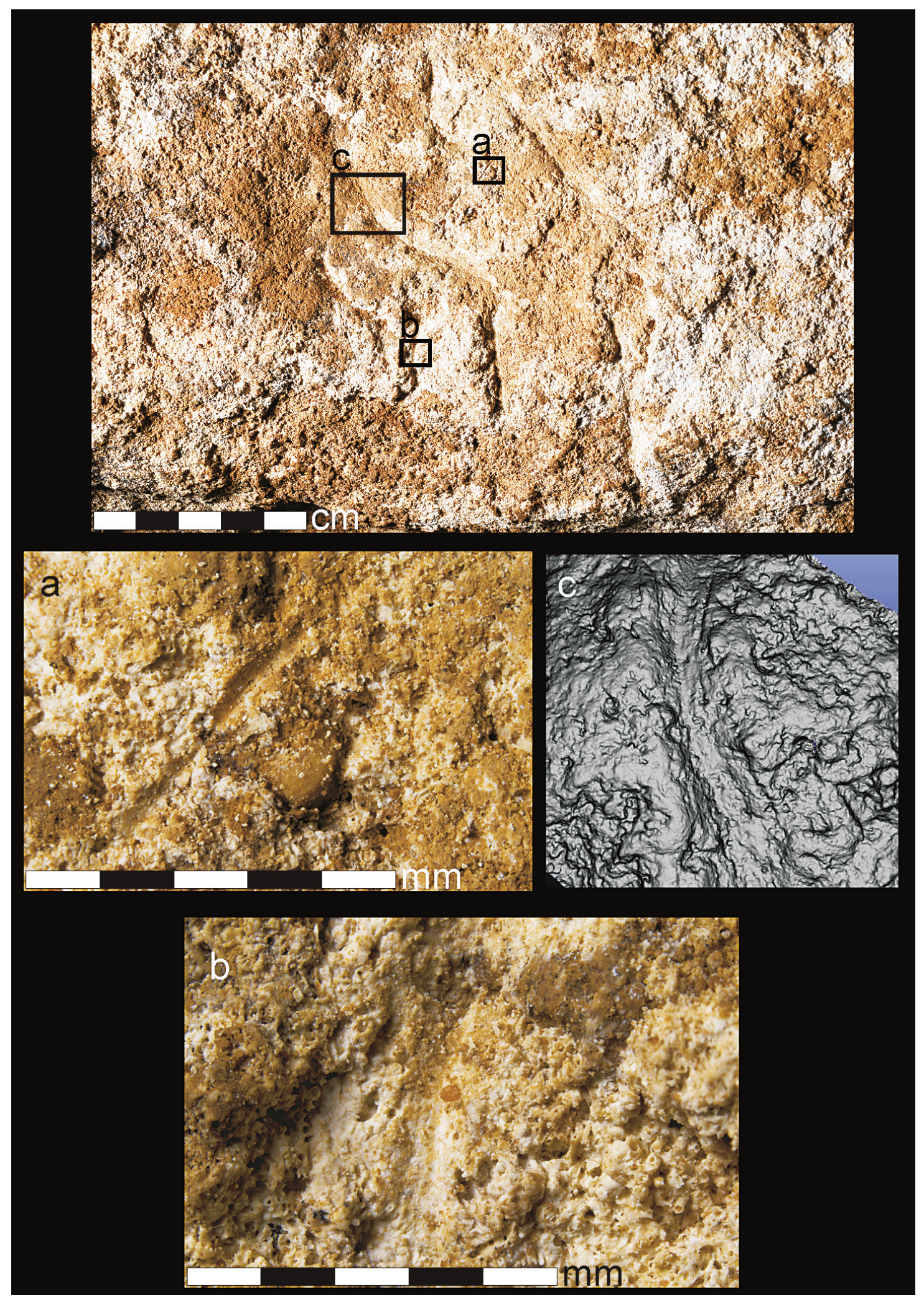

Figure 8 - Marques récentes datées du début du XXe siècle : a, b-Traces d'outil assez visibles; $c-3 D$ de l'une de ces marques avec la signature de l'outil sur les bords de la rainure (Photo L. Zotkina).

Figure 8 - Recent marks from the beginning of the XXth century: $a, b$ - tools marks quite visible; $c-3 D$ model of one of these modern traces with tool marks on the groove edges (Picture L. Zotkina). 
d'intensité inégale ayant, par endroits, surcreusé le tracé, le blanchissant à nouveau en lui donnant l'apparence de reprises avec une technique différente de celle utilisée par l'artiste paléolithique. Une analyse fine de ce tracé, à la loupe stéréoscopique, et des enregistrements de détails en 2 et 3 dimensions, ne révèlent que deux catégories de caractères : les unes relatives à la gravure profonde, ancienne, et les autres à la dégradation naturelle de la paroi. Aucun caractère ne peut être rapporté à du piquetage ni à aucune intervention plus récente sur la figure même, alors que la paroi montre, par ailleurs, des marques d'outils datant vraisemblablement des fouilles initiales dans la cavité.

Le regard tracéologique est partie intégrante de l'étude des représentations préhistoriques peintes. La problématique des reprises modernes, ou largement postérieures à leur réalisation initiale, porte ici sur les formes gravées, sculptées et piquetées ; elle ouvre un champ d'expertise supplémentaire à la tracéologie, que nous illustrons avec la figuration du bison de la grotte de La Grèze.

\section{Remerciements}

La présente étude a été réalisée grâce à une bourse du Centre d'étude franco-russe de Moscou et au soutien du Laboratoire international associé Artemir.

Nous remercions J.-J. Cleyet-Merle, directeur du Musée national de Préhistoire des Eyzies-de-Tayac, pour nous avoir permis de travailler dans la grotte de La Grèze, et G. Lévy, responsable de la gestion des grottes de Font-deGaume et de Combarelles, pour son aide logistique.

\section{Références bibliographiques}

AMPOULANGE M., PINTAUD R.C. 1955 - Une nouvelle gravure de la grotte de La Grèze, Bulletin de la Société préhistorique française, 53, p. 249-250

ARLES A., CLERC P., SARAH G., TÉREYGEOL F., BONNAMOUR G., HECKES J. et KLEIN A. 2013 - 3D reconstruction and modeling of subterranean landscapes in collaborative mining archaeology projects: techniques, applications and experiences. In: XXIV International CIPA Symposium, 2 - 6 September 2013, Strasbourg, France. I vol XL-5/W2. 2013 (International Archives of the Photogrammetry, Remote Sensing and Spatial Information Sciences).p. 61-66.

AUBRY T. et SAMPAIO J. D. 2008 - Fariseu: new chronological evidence for open-air Palaeolithic art in the Côa valley (Portugal). Antiquity, vol 82, $\mathrm{n}^{\circ} 316$. http://antiquity.ac.uk/ProjGall/aubry316/

AUBRY T, SANTOS A. T., LUIS L. 2014 - Stratigraphies du panneau 1 de Fariseu :analyse structurelle d'un système graphiquepaléolithique à l'air librede la vallée du Côa (Portugal), inPaillet P. (dir.), Les arts de la Préhistoire: micro-analyses, mises en contextes et conservation,Actes $\mathrm{du}$ colloque « Micro-analyses et datations de l'art préhistorique dans son contexte archéologique ", MADAPCA - Paris, 16-18 novembre 2011, Paleo, numéro spécial, p. 259-270

AUJOULAT N. 1984 - Grotte de La Grèze, L'art des cavernes. Atlas des grottes ornées paléolithiques françaises. Ministère de la Culture, Imprimerie Nationale, Paris, p. 164-166, 4 fig.

AVRILLAUD C. 2015 - Les saignées «naviformes» dans l'art rupestre postglaciaire : caractérisation morphologique et techno-tracéologique. Master 2 Anthropologie biologique - Préhistoire, Bordeaux : Université de Bordeaux, 69 p.

BELARBI M., RAYMOND P., SAULIĖRE N., TOUQUET R. 2012 - Acquisition 3D par photogrammétrie et illustration archéologique. JIAP. Livret des communications. Paris, L'Institut d'Art et d'Archéologie, p. 3.

BENSON L. V., HATTORI E. M., SOUTHON J. et ALECK B. 2013 - Dating North America's oldest petroglyphs, Winnemucca Lake subbasin, Nevada. Journal of Archaeological Science, 2013, vol 40, $n^{\circ} 12$, p. 4466-4476.

BREUIL H. 1952 - Quatre cents siècles d'art pariétal. Les cavernes ornées de l'âge du renne. Montignac, Centre d'Études et de documentation préhistoriques, p. 286-287.

CASSEN S., LESCOP L., CRIMAUD V., SUNER B. 2012 Bienfaits et limites d'un enregistrement lasergrammétrique dans la tombe à couloir de Gavrinis (Morbihan, France). JIAP. Livret des communications. Paris, L'Institut d'Art et d'Archéologie, p. 4.

CAPITAN L. BREUIL H., AMPOULANGE M. 1904a - Une nouvelle grotte préhistorique à parois gravées : la grotte de La Grèze (Dordogne), Comptes-rendus de l'Académie des Inscriptions et Belles-Lettres, 48 année, $n^{\circ}$ 5, p. 487-495.

CAPITAN L., BREUIL H., AMPOULANGE M. 1904b Nouveaux détails sur la grotte à parois gravées de La Grèze, Comptes-rendus des séances de l'Académie des Inscriptions et Belles-Lettres, 48ème année, n 5, p. 487-495.

CLOTTES J. 2010 - La grotte Chauvet : l'art d'origine. Seuil, $226 \mathrm{p}$.

CRETIN C., (dir.), avec la collab. de Armand D., Boche E., Bruxelles L., Cahoreau N., Chancerel A., Deneuve E., Genty D., Hoerlé S., Konik S., Le Fillâtre V., Lesvignes E., Madelaine S., Man-Estier E., Mangier E., Mensan R., Morala A., Muth X., Paillet P., Petrognani S., Plisson H., Robert É., Sisk M.L., Archambeau C., Bureau P., Couture C., Henriette C., Jugie Ph., Portais J.-C., VANZO J.-P., VEYRET M. 2015 - PCR Archéologie des sites ornés de Dordogne : cadre conceptuel, potentiels, réalité et opérations associées. Rapport 2014. Opération archéologique $n^{\circ}$ 2014-64. Bordeaux : Service régional de l'archéologie, $433 \mathrm{p}$.

CRETIN C. 2015 - Archéologie des sites ornés de Dordogne : cadre conceptuel, potentiels et réalité, rapport 
de Programme collectif de recherche du Ministère de la Culture et Communication, Opération archéologique $n^{\circ}$ 2014-64, 229 p.

DELLUC B. et DELLUC G. 1991 - L'art pariétal archaïque en Aquitaine, XXVIII supplément à Gallia Préhistoire, CNRS Ed., Paris, 390 p.

DELLUC B. et DELLUC G. 1992 - Grèze (La), La naissance de l'art en Europe, Union Latine, Paris, p. 281-282.

DELLUC B. et G., BOUVIER J.-M. 1984 - Deux nouvelles gravures dans la grotte de La Grèze, Bulletin de la SHAP, 111, p. 253-259.

D'ERRICO F., DAYET BOUILLOT L., GARCIA-DIEZ M., PITARCH MARTI A., GARRIDO PIMENTEL D. et ZILHÃO J. 2016 - The technology of the earliest European cave paintings: El Castillo Cave, Spain. Journal of Archaeological Science, 2016, vol 70, $\mathrm{n}^{\circ}$ June, p.48-65.

D'ERRICO F., SACCHI D. et VANHAEREN M. 2002 L'analyse technique de l'art gravé de Fornols-Haut, Campôme, France. Implications dans la datation des représentations de style paléolithique à l'air libre. In : Sacchi D. dir, L'art paléolithique à l'air libre : le paysage modifié par l'image, Tautavel-Campôme, 7-9 octobre 1999. Carcassonne, Gaep \& Géopré, p. 75-86.

GENTY D., KONIK S., VALLADAS H., BLAMART D., HELLSTROM J., TOUMA M., MOREAU C., DUMOULIN J-P., NOUET J., DAUPHIN Y.,WEIL R. 2011 Dating the Lascaux cave gours formation. Radiocarbon, Volume 53, $n^{\circ} 3, p$ 479-500.

HINOUT J. 1998 - Les pétroglyphes mésolithiques des massifs gréseux du Bassin parisien. Revue archéologique de Picardie, vol 3, $n^{\circ} 1$, p. 31-52.

JAUBERT J. 2008 - L'« art » pariétal gravettien en France : éléments pour un bilan chronologique, Le Gravettien : entités régionales d'une paléoculture européenne, Table ronde - Les Eyzies - Juillet 2004, Les Eyzies-de-Tayac, Paleo, 20, p. 439-474, 27 fig., 2 tabl.

KERVAZO B., FERUGLIO V., BAFFIER D., DEBARD É., FERRIER C., PERROUX A.-S., AUJOULAT N., DELANNOY J.-J. et PERRETTE Y. 2010 - Paroi et art pariétal : approche taphonomique. L'exemple de la grotte Chauvet-Pont d'Arc (Ardèche), Paleo Supplément $\mathrm{n}^{\circ} 4$ septembre 2010, Mise en commun des approches en taphonomie - IUSPP - Lisbonne - 2006, p. 43-52

LONGO L. et SKAKUN N. 2005 - The roots of use-wear analysis: selected papers of S.A. Semenov. Memorie del Museo Civico di Storia Naturale di Verona (2 serie). Sezione Scienze Dell'Uomo, 7. Verona: Museo Civico di Storia Naturale - Verona, $141 \mathrm{p}$.

MIKLASHEVICH E. 2008 - Rock Art Research in Siberia and Central Asia, 2000-2004, Rock Art Studies. News of the World, III, Oxbow Books, Oxford, UK, pp. 138-179.
PEYRONY D. 1949 - La grotte de La Grèze, commune de Marquay, Bull. Soc. Hist. Arch. Périgord, 76, pp. 53-58, 1 fig.

PLETS G., GHEYLE W., VERHOEVEN G., DE REU J., BOURGEOIS J., VERHEGGE J., STICHELBAUT B. 2012 Three-dimensional recording of archaeological remains in the Atlai Mountains. Antiquity Publications, ${ }^{\circ}$ 86. p. 884-897.

PLISSON H. 2015 - Digital photography and traceology: from 2D to 3D. Traces in the history. Dedicated to 75 anniversary of Viacheslav E. Shchelinsky. / dir par Lozovskaya O.V., Lozovski V.M. et Girya E. Yu. Saint Petersbourg : IIMKRAN, p. 272.

PLISSON H. et ZOTKINA L. V. 2015 - From 2D to 3D at macro- and microscopic scale in rock art studies. Digital Applications in Archaeology and Cultural Heritage, vol 2, $\mathrm{n}^{\circ} 2-3$, p. 102-119.

RIGAUD J.-P. 1984 - Grotte de La Grèze, Gallia Préhistoire, Informations archéologiques : circonscription d'Aquitaine, t. 27, fasc. 2, p. 278.

ROBERT E., EGELS Y., BOCHE E., VIGEARS D., VIALOU D. 2012 - La photogrammétrie en grotte ornée : application pour l'étude et la contextualisation de l'art préhistorique dans les grottes Blanchard (Indre) et Rouffignac (Dordogne). JIAP. Livret des communications. Paris, L'Institut d'Art et d'Archéologie, p. 26.

ROUSSOT A. 1965 - Les découvertes d'art pariétal en Périgord, Centenaire de la Préhistoire en Périgord (18641964), supplément au Bulletin de la SHAP, Périgueux, t. 91, p. 115.

SEMENOV S. A. 1964 - Prehistoric technology; an experimental study of the oldest tools and artefacts from traces of manufacture and wear. London: Cory, Adams \& Mackay, 1964 - $211 \mathrm{p}$.

SONNEVILLE-BORDES D. (de) 1960 - Le Paléolithique supérieur en Périgord, Thèse de doctorat, Delmas Imp., Périgueux, p. 321, 1 fig. et pp. 427-428, 1 fig.

SONNEVILLE-BORDES D. (de) 1965 - Les industries des abris et grottes ornées du Périgord. Centenaire de la Préhistoire en Périgord, 1864-1964, n spécial du Bulletin de la Société Historique et Archéologique du Périgord, p. 176.

TOSELLO G. et FRITZ C. 2005 - Les dessins noirs de la grotte Chauvet-Pont d'Arc : essai sur leur originalité dans le site et leur place dans l'art aurignacien. Bulletin de la Société Préhistorique Française, vol 102, n 1, p. 159-171

VIDAL P. 1967 - Grottes et abris ornés de la vallée de la Vézère (Dordogne), Spelunca Bulletin, 3, p. 194-201, 3 fig.

ZOTKINA L. V. 2012 - Études technologiques des pétroglyphes de Sibérie (site de Shalobolino, région de Krasnoyarsk). In : J. Clottes dir, L'art pléistocène dans le monde. Actes du Congrès IFRAO. Tarascon-sur-Ariège, 
septembre 2010. Société Préhistorique Ariège-Pyrénées, p. $189-190$.

ZOTKINA L. V., TEKHTEREKOV A. S., KHAREVITCH V. M. et Plisson H. 2014 - An Experimental Study of Percussion Technologies in the Minusinsk Basin: Percussion and Tool Types. Archaeology, Ethnology and Anthropology of Eurasia, vol 42, n¹, p. 55-65.

ГИРЯЕ.Ю., ДЭВЛЕТЕ.Г. 2010 - Quelques résultats de la recherche méthodologique dans l'étude de la technique de piquetage en art rupestre (Некоторыерезультатыразработкиметодикиизучениятехникивыполненияпетр оглифовпикетажем). Уральскийисторическийвестник, $\mathrm{n}^{\circ} 1$ (26), Екатеринбург, р. 107-118.

МИКЛАШЕВИЧЕ. А. 2011 - À l'étude de la technique d'exécution des représentations de Tomskayapissanitsa ( изучениютехникинанесенияизображенийТомскойписани цы).Историко-культурное наследие Кузбасса (актуальные проблемы изучения и охраны памятников археологии), III, Кузбассиздат, Кемерово, Россия, р. 132-155.
CEMEHOB C.A.1957 - Technologie préhistorique (Первобытная техника). Материалы и исследования по Археологии СССР, 54. Москва, Ленинград: Академия Наук СССР, 1957, 240p.

ЗОТКИНА Л.В. 2013 - Pétroglyphes piquetés du bassin de Minoussinsk (aux matérieux de la recherche technotracéologique) (Петроглифы Хакасско-Минусинской котловины, выполненные в технике пикетажа (по данным технолого-трасологического исследования)). Кандидатская диссертация. Археология, Новосибирск: Новосибирский государственный университет, 304 р.

ЧЕРЕМИСИН Д.В., ЗОТКИНА Л.В., МИКЛАШЕВИЧ Е.А., ЛБОВА Л.В., ЖЕНЕСТ Ж.-М., ПЛИССОН Ю.,КРЕТАНК. 2013 - Étude technologique de l'art rupestre des monts d'Altaï en 2013 (Исследование технологических особенностей наскальных изображений горного Алтая в 2013 году). Материалы Итоговой сессии Института археологии и этнографии СО PAH, vol. XIX, p. 362-368. 